Revista Brasileira de Agricultura Irrigada v.12, nº.5, p. 2866 - 2876, 2018

ISSN 1982-7679 (On-line)

Fortaleza, CE, INOVAGRI - http://www.inovagri.org.br

DOI: $10.7127 /$ rbai.v12n500847

Protocolo 847.18 - 20/12/2017 Aprovado em 30/09/2018

\title{
ANÁLISE ESPAÇO-TEMPORAL DA EVAPOTRANSPIRAÇÃO DE REFERÊNCIA PARA O ESTADO DO MARANHÃO
}

Jhonata Santos Santana ${ }^{1}$, Wilson Araújo da Silva², Elton Ferreira Lima ${ }^{3}$, Gustavo Costa de Oliveira $^{4}$

\section{RESUMO}

A evapotranspiração de referência (ETo) é uma variável relevante para o planejamento e manejo da irrigação, pois permite quantificar a demanda de água em uma determinada área de cultivo, informação esta, de fundamental importância no manejo adequado da irrigação. Assim esse trabalho teve como objetivo caracterizar a distribuição espacial e temporal da Evapotranspiração de referência-ETo para o Estado do Maranhão e gerar informações que possam auxiliar os técnicos e produtores rurais no dimensionamento e manejo de sistemas de irrigação para distintas regiões do Estado. Os valores de ETo foram estimados pelo método de Penman-Monteith-FAO e espacializados utilizando-se o método de interpolação IDW. Através dos mapas interpolados de ETo verificou-se que o trimestre mais seco é formado pelos meses de agosto, setembro e outubro e as médias mensais de ETo variaram de 2,05 mm dia-1 (fevereiro) à 3,86 mm. dia ${ }^{-1}$ (setembro). A região Leste (na divisa com Piauí) é a que apresenta maior de déficit hídrico ao longo do ano. Percebe-se que nas Regiões Sudoeste e Sul a ocorrência de maiores valores de ETo nos meses de maio a setembro caracterizando o pico da estação seca nessas regiões. Já período que vai de outubro a fevereiro é caracterizado por uma clara inversão de padrões de ETo ocorrendo o pico da estação seca nas Regiões Norte, Leste e Central do Maranhão.

Palavras-chave: Irrigação, Penman-Monteith-FAO, interpolação

\section{SPACE-TEMPORAL ANALYSIS OF EVAPOTRANSPIRATION OF REFERENCE FOR THE STATE OF MARANHÃO}

\footnotetext{
ABSTRACT

The reference evapotranspiration (ETo) is a relevant variable for the planning and management of irrigation, since it allows quantifying the water demand in a given area of crop, which is of

${ }^{1}$ Graduado em Engenharia Agronômica, Bolsista BATI-II UEMASUL, Imperatriz - Maranhão, Email: agro.starf@gmail.com

${ }^{2}$ Prof. Doutor UEMASUL, campus Imperatriz- Maranhão, Email: wilsonuemasul@gmail.com

${ }^{3}$ Graduando do curso de Engenharia Agronômica, UEMASUL, campus Imperatriz-Maranhão

${ }^{4}$ Mestrando em Agricultura e Ambiente, UEMA, campus São Luís-Maranhão
} 
fundamental importance in the proper management of irrigation. The objective of this work was to characterize the spatial and temporal distribution of ETo reference evapotranspiration for the State of Maranhão and to generate information that may help technicians and rural producers in the design and management of irrigation systems for different regions of the State. The ETo values were estimated by the Penman-Monteith-FAO method and spatialized using the IDW interpolation method. The ETo interpolated maps showed that the driest quarter is formed by the months of August, September and October and the monthly averages of ETo ranged from $2.05 \mathrm{~mm}$ day-1 (February) to $3.86 \mathrm{~mm}$. day-1 (September). The eastern region (on the border with Piauí) is the one with the highest water deficit during the year. It can be seen that in the Southwest and South regions, the highest ETo values occurred in the months of May to September, characterizing the dry season peak in these regions. The period between October and February is characterized by a clear inversion of ETo patterns, with the peak of the dry season in the North, East and Central Regions of Maranhão.

Keywords: Irrigation, Penman-Monteith-FAO, interpolation

\section{INTRODUÇÃO}

A determinação das necessidades hídricas dos cultivos é uma preocupação básica e de fundamental importância, pois trata-se de um parâmetro de referência na realização do planejamento e operação de sistemas de irrigação, sobretudo quanto se busca a otimização dos recursos hídricos disponíveis (TABARI et al., 2013).

Informações sobre a evapotranspiração de referência (ETo) são ferramentas importantes no manejo da irrigação, pois são utilizadas tanto por organizações que gerenciam sistemas de distribuição de água para irrigação, visando à avaliação da eficiência e melhoria de suas capacidades, quanto por agricultores que desejam conhecer as demandas de água específicas de um cultivo (SILVA et al., 2012).

A FAO (Organização das Nações Unidas para Agricultura e Alimentação) e a ICID (International Commission of Irrigation and Drainage) adotaram o método de PenmanMonteith como padrão da FAO (Boletim 56) para a estimativa da Evapotranspiração de referência (ETo), devido a sua formulação física e pelos excelentes resultados obtidos nas mais distintas condições climáticas (BERNARDO et al., 2005; MARTÍ et al., 2015). O método Penman-Monteith-FAO (PM-FAO) é considerado, internacionalmente, $\mathrm{o}$ mais apropriado para a estimativa da ETo, pois, além de procurar representar de maneira consistente o fenômeno biofísico da evapotranspiração, é alimentado por quase todos os elementos meteorológicos observados em estações meteorológicas de superfície (CARVALHO et al., 2011). Este método tem sido aplicado com sucesso em diferentes condições agrometeorológicas, sendo considerado robusto e preciso (RÁCZ et al., 2013).

O conhecimento das necessidades hídricas dos cultivos juntamente com análises de sua distribuição espacial e temporal possui aplicação em várias etapas do processo de planejamento e gerenciamento dos recursos hídricos, sendo também uma variável utilizada em vários outros estudos agrometeorológicos (USMAN et al., 2015). Diante disso os Sistemas de Informação Geográfica (SIG), vêm sendo adotados em grande parte dos projetos que envolvem a utilização de dados espaciais, permitindo-se estudar componentes ambientais de forma isolada ou integrada (MENESES; NASCIMENTO, 2014). O uso do SIG na agrometeorologia visa solucionar a ausência de informações regionalizadas, pois uma das aplicações principais do SIG em agrometeorologia é a de transformar dados numéricos, obtidos em pontos referenciados geograficamente na superfície, em mapas interpolados, obtendo-se valores estimados para todas as localidades da região representada, que resulta numa série de informações confiáveis a respeito do comportamento espacial da variável, sem a necessidade de observação direta Pellegrino et al., (1998). 
De acordo com Vilanova et al., (2012) a distribuição de variáveis como a ETo e a precipitação em mapas interpolados facilitam a análise espacial e temporal, tornando-a clara e objetiva, auxiliando quem deseja ter conhecimento sobre estas informações, permitindo fácil entendimento e visualização.

A representação da evapotranspiração de referência (ETo) em mapas interpolados pode contribuir para um adequado planejamento e manejo racional de sistemas de irrigação, em distintas regiões do Estado do Maranhão. Diante disso, o trabalho teve como objetivo interpolar dados de evapotranspiração de referência a fim de caracterizar a distribuição espacial e temporal da ETo para o estado do Maranhão e gerar informações que possam auxiliar os técnicos e produtores rurais no dimensionamento e manejo de sistemas de irrigação para distintas regiões do Estado.

\section{MATERIAL E MÉTODOS}

Antes da escolha do método de interpolação adequado a situação de estudo foram realizados testes de ajuste para equações lineares, quadráticas e exponenciais, entretanto devido a quantidade reduzida de estações meteorológicas automáticas do estado do Maranhão, não foram obtidos ajustes satisfatórios para nenhum dos modelos testados, que resultaria no emprego do método Krigagem de interpolação, nessas situações em que não há ajuste de equações é recomendado o emprego do método de interpolação IDW (Inverse Distance Weighting). Entretanto, este estudo é um passo importante para o sucesso da agricultura irrigada no estado do Maranhão, uma vez que busca esclarecer dúvidas antigas feitas por técnicos e pesquisadores sobre o comportamento espaço temporal da ETo no estado. Vale ressaltar, que maioria dos praticantes da agricultura irrigada no estado, não possuem qualquer noção teórica, sobre quantidade de água a aplicar, que é muitas vezes baseada no conceito prático do agricultor, o que pode acarretar déficit ou excesso hídrico para as culturas (FARIA et al., 2012). Assim, este estudo busca fornecer informações que poderão servir como ponto de partida, na investigação sobre as reais necessidades hídricas das culturas para distintas regiões do Estado.

\section{Caracterização das estações meteorológicas utilizadas no estudo}

Para obtenção da demanda de irrigação da cultura de referência nas distintas regiões do Estado foram utilizados dados de 43 estações automáticas meteorológicas ligadas à rede do INMET (Instituto Nacional de Meteorologia), sendo que 14 pertencem ao Estado do Maranhão, 09 ao Estado do Pará, 08 do Estado do Tocantins e 12 do Estado do Piauí. A seleção de estações fora do Estado do Maranhão se dá para fins de melhoria na interpolação dos dados por meio do SIG utilizado no trabalho, conforme a metodologia adotada por Carvalho et al., (2006).

A seleção das estações foi baseada na disponibilidade de dados climáticos e na sua distribuição espacial, assim adotou-se séries históricas variadas de acordo com a data de fundação das estações referentes à umidade relativa média do ar, velocidade do vento, temperatura máxima, temperatura mínima, longitude, latitude, altitude e outras informações necessárias para a aplicação do método de Penman-Monteith-FAO (PM-FAO).

$\mathrm{O}$ processo utilizado para o preenchimento de falhas, isto é, ausências de dados utilizados para o cálculo da ETo pelo método de Penman-Monteith-FAO, foi rigorosamente observado e foi estabelecido o padrão recomendado no boletim da FAO N 56 de 1998, ou seja, para os dias com ausência de dados no mês, foi considerada a média do mês em questão para o preenchimento da falha. $\mathrm{Na}$ Tabela 1 estão apresentadas as estações meteorológicas automáticas que foram utilizadas no estudo. 
Tabela 1. Código, nome, latitude, longitude e altitude das estações meteorológicas utilizadas

\begin{tabular}{|c|c|c|c|c|c|}
\hline Código & Nome & $\begin{array}{c}\text { Latitude } \\
\text { (S) }\end{array}$ & $\begin{array}{c}\text { Longitude } \\
\text { (W) }\end{array}$ & $\begin{array}{c}\text { Altitude } \\
\text { (m) }\end{array}$ & Período \\
\hline 81985 & Alto- Parnaíba-MA & -0906 & -4555 & 284 & 2008-2016 \\
\hline 81790 & Bacaba-MA & -0414 & -4447 & 22 & 2008-2016 \\
\hline 81903 & Balsas-MA & -0727 & -4601 & 271 & 2007-2016 \\
\hline 81901 & Carolina-MA & -0720 & -4727 & 183 & 2007-2016 \\
\hline 81749 & Chapadinha-MA & -0344 & -4321 & 104 & 2008-2016 \\
\hline 81866 & Colinas-MA & -0601 & -4414 & 178 & 2008-2016 \\
\hline 81822 & Imperatriz-MA & -0533 & -4745 & 118 & 2008-2016 \\
\hline 81715 & São-Luís-MA & -0231 & -4412 & 55 & 2003-2016 \\
\hline 81687 & Turiçú-MA & -0139 & -4522 & 36 & 2008-2016 \\
\hline 81788 & Buriticupu-MA & -0419 & -4626 & 175 & 2008-2016 \\
\hline 81863 & Estreito-MA & -0639 & -4725 & 173 & 2008-2016 \\
\hline 81716 & Farol_de_Santana-MA & -0216 & -4337 & 10 & 2008-2016 \\
\hline 81823 & Grajaú-MA & -0548 & -4609 & 232 & 2008-2016 \\
\hline 81717 & Farol de Preguiças-MA & -0235 & -4242 & 5 & 2008-2016 \\
\hline 81685 & Bragança-PA & -0104 & -4646 & 41 & 2008-2016 \\
\hline 81684 & Capitão_Poço-PA & -0144 & -4703 & 79 & 2011-2016 \\
\hline 81820 & Marabá-PA & -0510 & -4922 & 117 & 2009-2016 \\
\hline 81745 & Paragominas-PA & -0300 & -4720 & 113 & 2007-2016 \\
\hline 81784 & Novo_Repartimento-PA & -0415 & -4955 & 101 & 2008-2016 \\
\hline 81786 & Rondon_do_Pará-PA & -0447 & -4804 & 207 & 2008-2016 \\
\hline 81860 & Serra_dos_Carajás-PA & -0604 & -5008 & 707 & 2008-2016 \\
\hline 81711 & Tomé_Açú-PA & -0224 & -4809 & 43 & 2007-2016 \\
\hline 81743 & Tucuruí-PA & -0349 & -4940 & 138 & 2008-2016 \\
\hline 81846 & Alvorada_do_Gurg.-PI & -0826 & -4351 & 261 & 2007-2016 \\
\hline 81752 & Parnaíba-PI & -0305 & -4147 & 52 & 2003-2016 \\
\hline 81987 & Bom_Jesus_do_Piaui-PI & -0905 & -4419 & 296 & 2007-2016 \\
\hline 81847 & Canto_do_Buriti-PI & -0807 & -4258 & 312 & 2010-2016 \\
\hline 81751 & Esperantina-PI & -0354 & -4214 & 88 & 2007-2016 \\
\hline 81986 & Gilbues_PI & -0952 & -4520 & 425 & 2009-2016 \\
\hline 81869 & Oeiras-PI & -0658 & -4208 & 154 & 2008-2016 \\
\hline 81794 & Piripiri-PI & -0415 & -4146 & 158 & 2007-2016 \\
\hline 81828 & São_Pedro_do_Piaui-PI & -0554 & -4243 & 296 & 2009-2016 \\
\hline 81827 & Teresina-PI & -0504 & -4248 & 75 & 2005-2016 \\
\hline 81905 & Uruçuí-PI & -0728 & -4420 & 399 & 2008-2016 \\
\hline 81870 & Valença-PI & -0623 & -4144 & 313 & 2009-2016 \\
\hline 81900 & Araguaína-TO & -0706 & -4812 & 231 & 2007-2016 \\
\hline 81821 & Araguatins-TO & -0538 & -4806 & 131 & 2009-2016 \\
\hline 81902 & Campos_Lindos-TO & -0809 & -4638 & 427 & 2012-2016 \\
\hline 86632 & Dianopolís-TO & -1135 & -4650 & 728 & 2008-2016 \\
\hline 86608 & Mateiros-TO & -1025 & -4555 & 791 & 2012-2016 \\
\hline 86607 & Palmas-TO & -1011 & -4818 & 292 & 2004-2016 \\
\hline 81941 & Pedro_Afonso-TO & -0858 & -4811 & 190 & 2007-2016 \\
\hline 81981 & Rio_Sono_TO & -0916 & -4713 & 291 & 2016-2016 \\
\hline
\end{tabular}

FONTE: Próprio Autor.

Por meio do software Microsoft Office Excel ${ }^{\circledR}$ (2010) foram calculadas as estimativas da evapotranspiração de referência pelo método de Penman-Monteith-FAO (EToPM) para todas essas localidades (ALLEN et al., 1998). De posse dos valores de ETo que foram obtidos para as distintas localidades do Estado do Maranhão e os valores pertencentes aos Estados do Tocantins e Pará, foi possível extrapolar para as demais regiões do Estado utilizando o software livre de SIG QuantunGis ${ }^{\circledR}$ (versão 2.18-Las Palmas) conhecido popularmente como Qgis, que interpolou a médias de Evapotranspiração das séries históricas de dados climáticos. Os valores de Evapotranspiração de referência foram especializados utilizando-se o método de interpolação geoestatístico conhecido como interpolador linear IDW (Inverse Distance Weighted - Ponderação do Inverso da Distância), método baseado na dependência 
espacial, este modelo supõe que quanto mais próximo estiver um indivíduo do outro, maior será a correlação desse indivíduo com seus vizinhos, atribuindo-se maior peso para os indivíduos mais próximos do que para os mais distantes do ponto a ser interpolado, sendo uma das técnicas de interpolação mais usadas para pontos espalhados espacialmente (LI; HEAP, 2011).

\section{RESULTADOS E DISCUSSÃO}

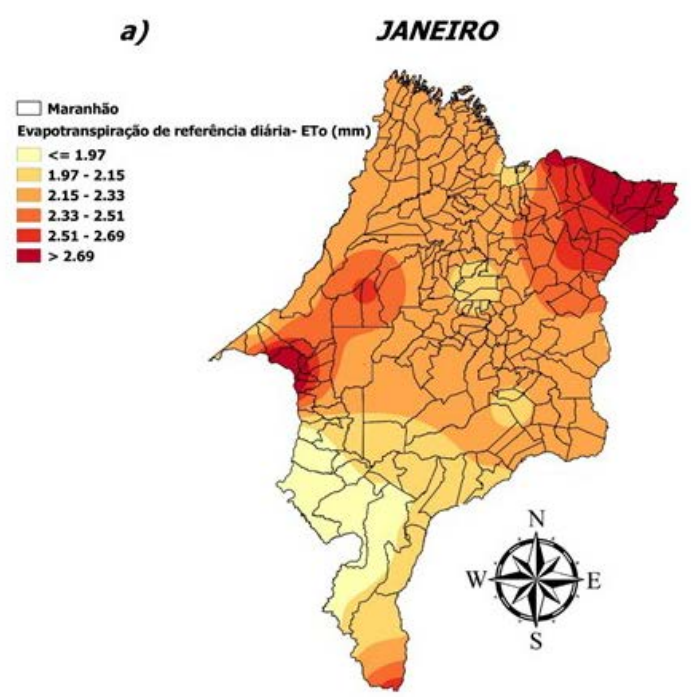

\section{Espacialização dos principais elementos meteorológicos utilizados no estudo}

Nas Figuras 1 a 6 apresentam-se a evapotranspiração de referência média diária espacializada para o Estado do Maranhão a partir da interpolação dos dados de 43 estações climatológicas, em que 14 pertencem ao Estado do Maranhão, 09 ao Estado do Pará, 08 do Estado do Tocantins e 12 do Estado do Piauí utilizando o interpolador geoestatístico IDW (Inverse Distance Weighted) adotando séries históricas variadas de dados climáticos de acordo com a disponibilidade em cada estação meteorológica.

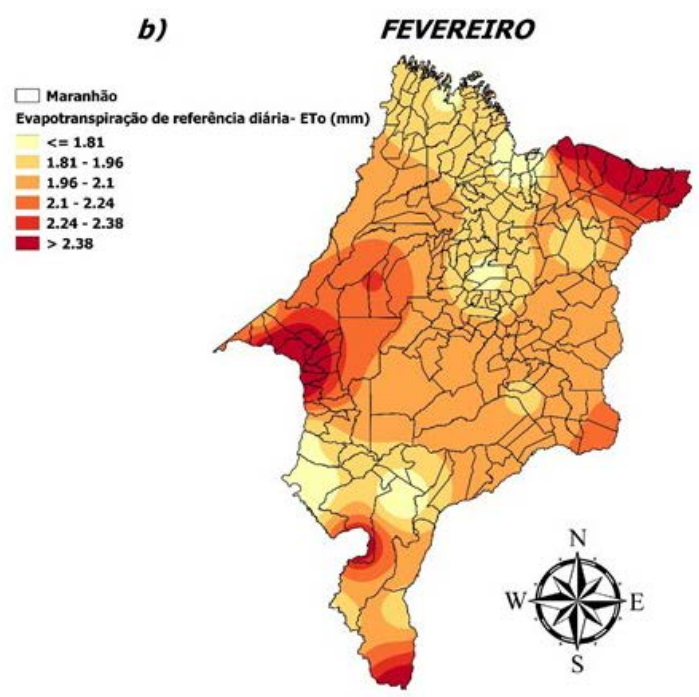

Figura 1. Evapotranspiração de referência para o Estado do Maranhão (a) janeiro (b) fevereiro
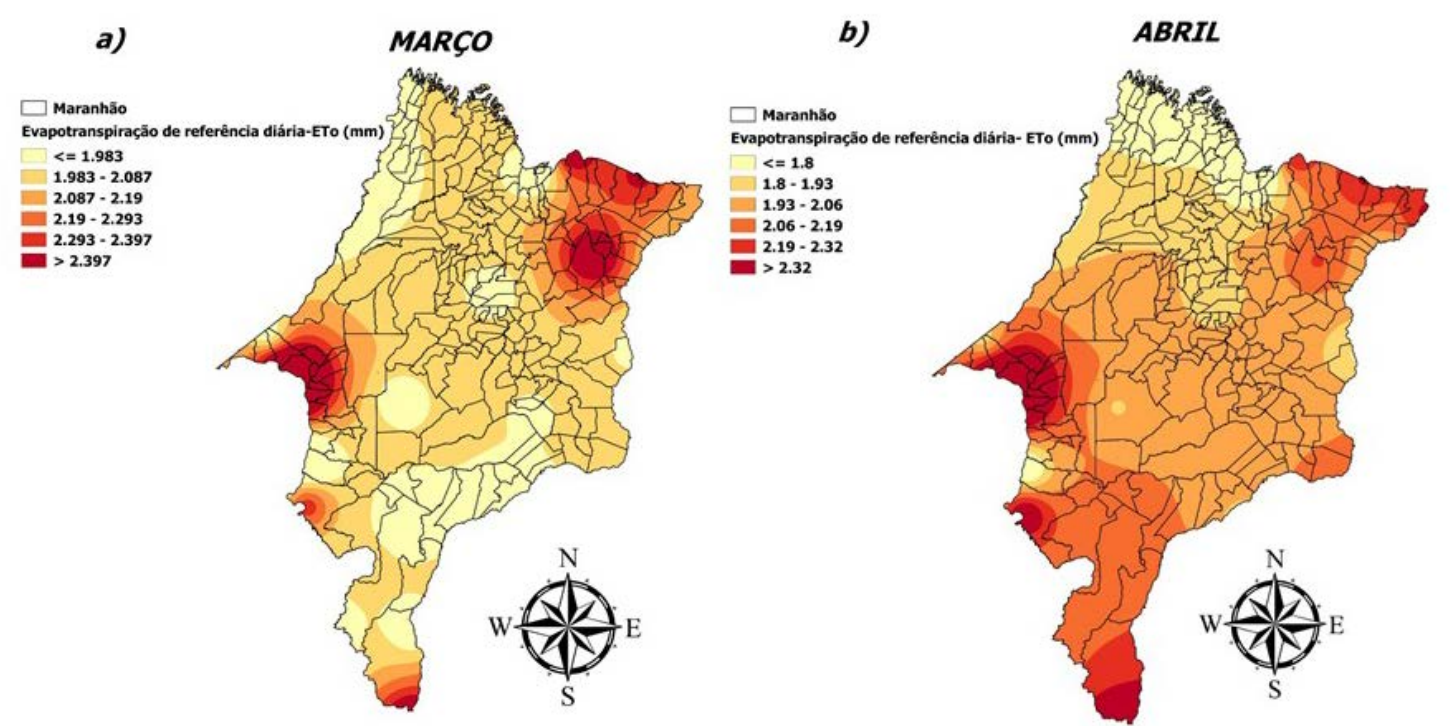

Figura 2. Evapotranspiração de referência para o Estado do Maranhão (a) março (b) abril. 
Santana et al.
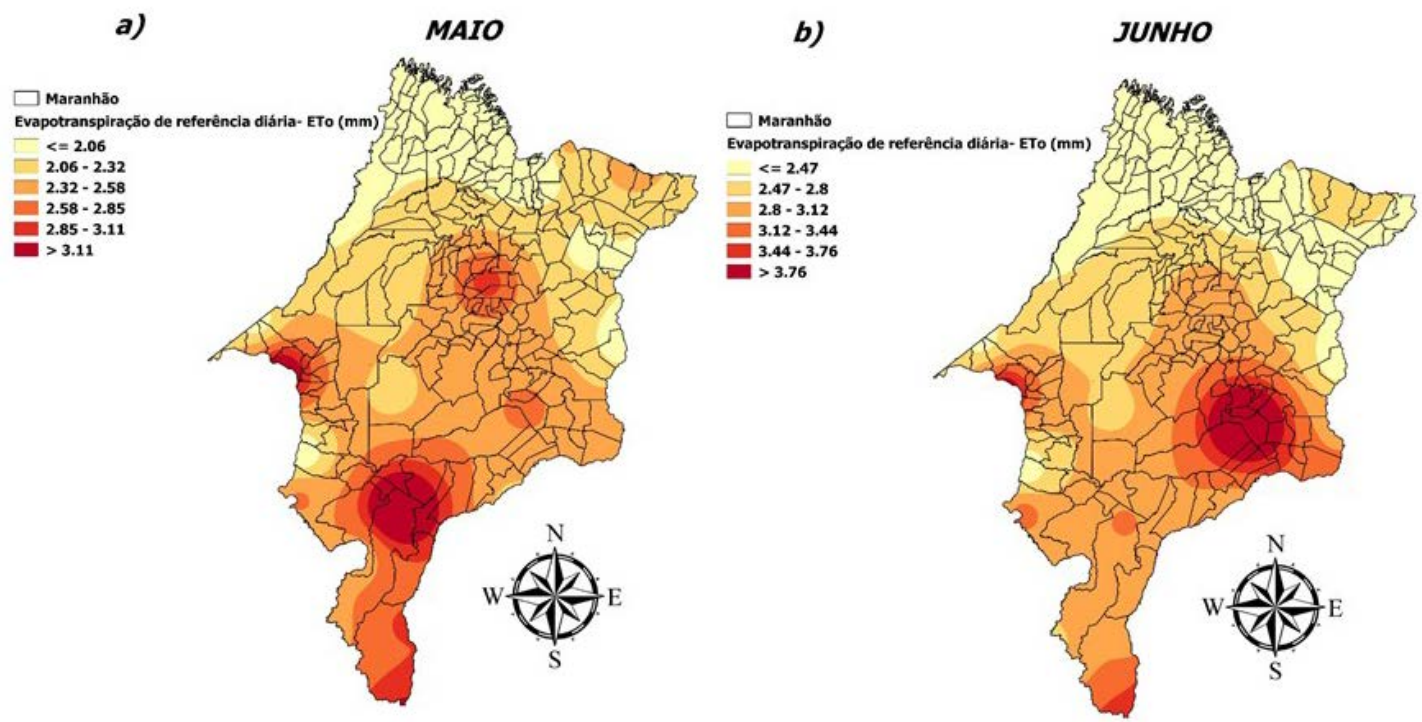

Figura 3. Evapotranspiração de referência para o Estado do Maranhão (a) maio (b) junho
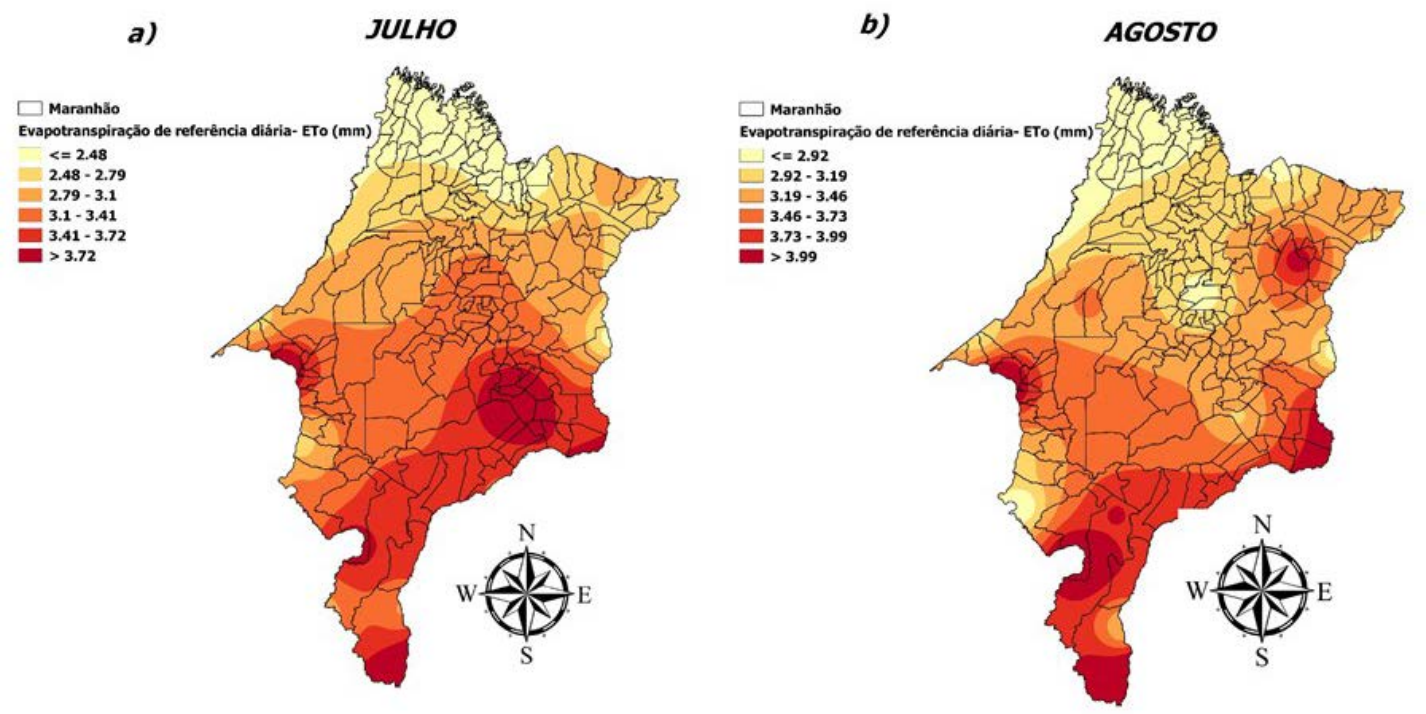

Figura 4. Evapotranspiração de referência para o Estado do Maranhão (a) julho (b) agosto
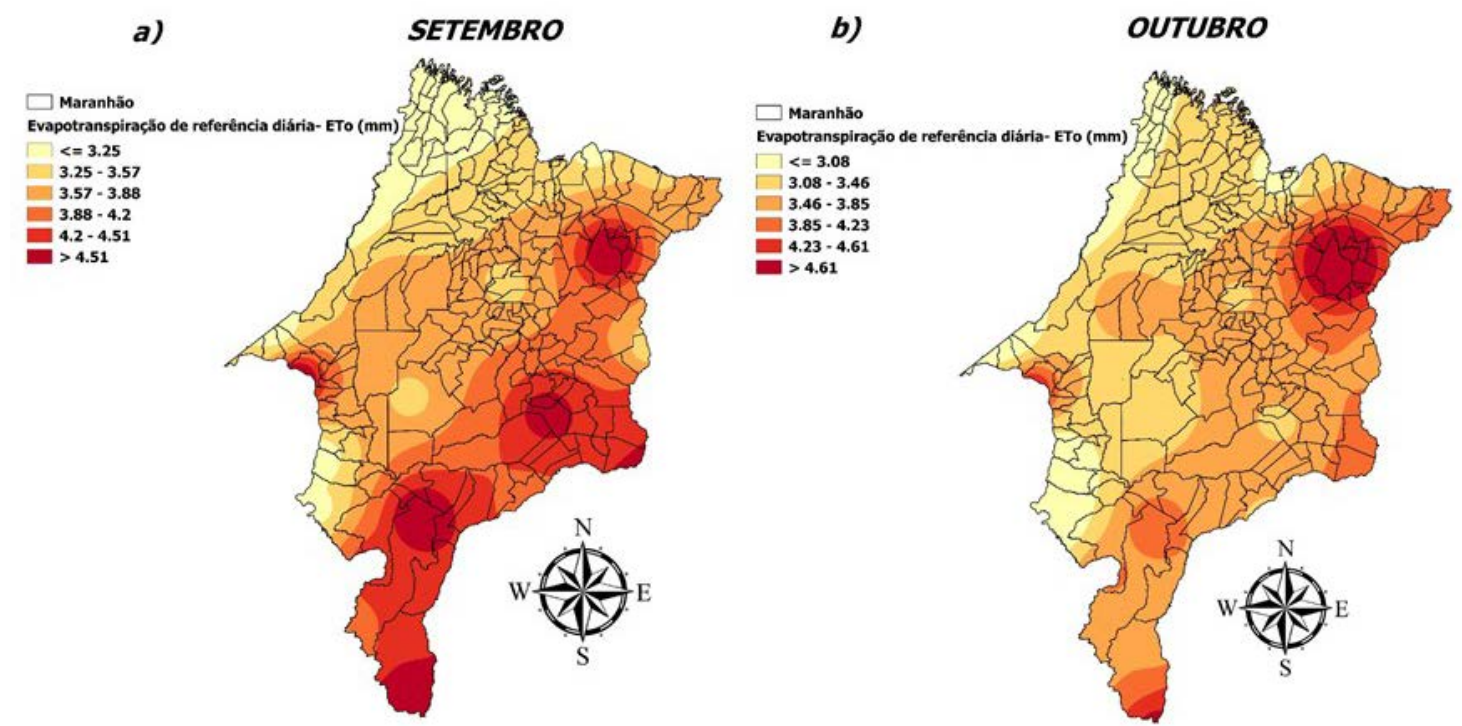

Figura 5. Evapotranspiração de referência para o Estado do Maranhão (a) setembro (b) outubro 

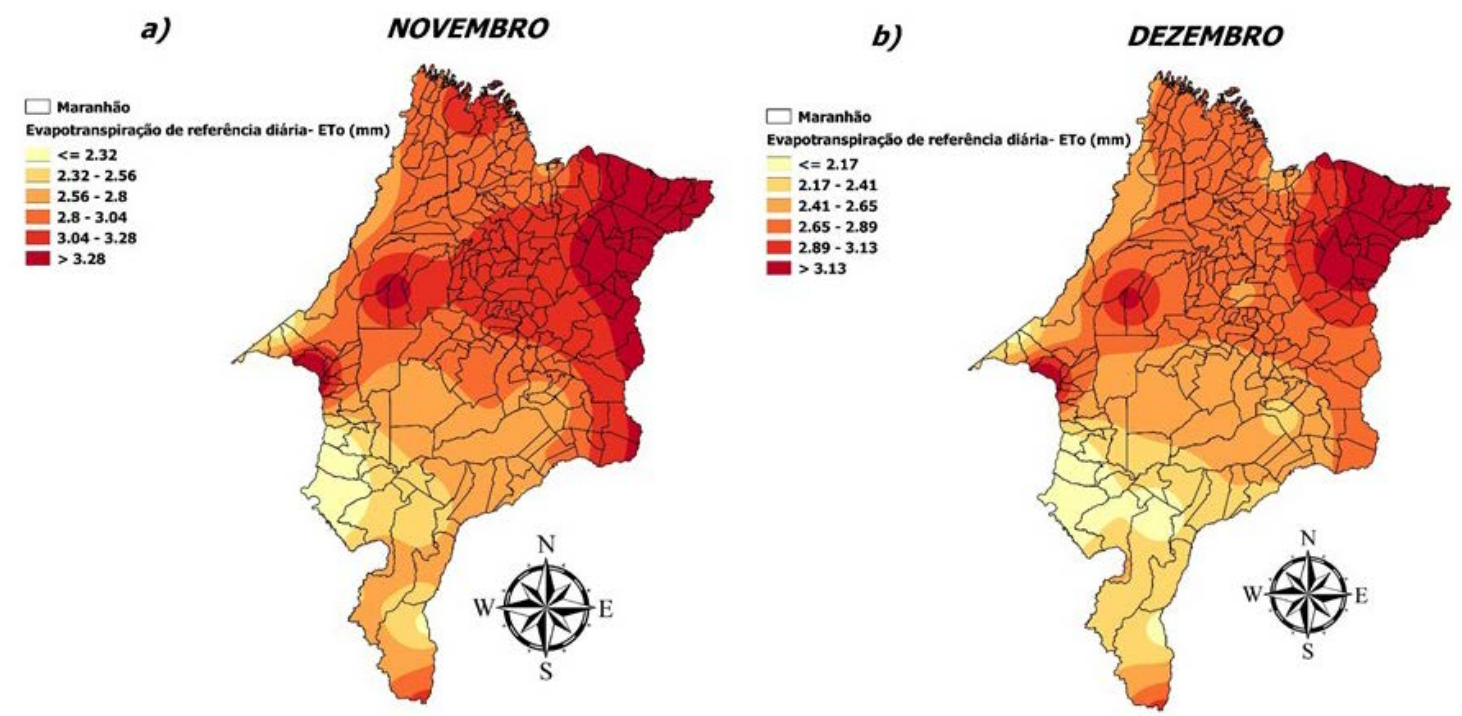

Figura 6. Evapotranspiração de referência para o Estado do Maranhão (a) novembro (b) dezembro

A Figura 1 (a), destaca a distribuição espacial da ETo para janeiro, mês que corresponde ao início da estação chuvosa no Maranhão. Nota-se que no mês de janeiro os valores mais elevados de ETo se concentram no Norte, Leste, Oeste e Centro maranhense com ocorrência de maior parte das áreas apresentando valores de ETo acima de 2,15 mm. dia $^{-1}$, há ocorrência pontuais de áreas com maiores valores nas regiões Litorâneas correspondente aos municípios de Tutóia, na região Oeste (municípios de Imperatriz e Buriticupu) e em áreas do extremo Leste (município de Araioses) e Leste (Regiões de divisa com Piauí). Sousa et al., (2015) obtiveram a média de 3,8 mm. dia ${ }^{-1}$ de ETo pelo método de Penman-Monteith FAO 56 para o mês de janeiro na região de transição Cerrado-Amazônica. Esse padrão de comportamento da ETo no norte do Estado, demostra que mês de janeiro corresponde a estação seca na maior parte da região Oeste, Centro e Norte do Estado e também a pequena influência da alta umidade relativa do ar que é característica da região litorânea na redução da ETo, ficando limitada apenas as áreas da Baixada correspondente a Região Metropolitana de São Luís. Pois quanto maior a umidade relativa do ar menor será o poder evaporante do ar, e, consequentemente menor será a ETo (TAGLIAFERRE et al., 2015).
A Figura 1 (b) apresenta a distribuição espacial distribuição espacial da ETo para o mês de fevereiro. Observa-se uma redução acentuada da ETo no Norte, Centro e Sul maranhense em consequência do início da estação chuvosa, o que contribui para a ocorrência de extensas áreas com valores abaixo de 1,81 mm. dia ${ }^{-1}$, caracterizando uma menor demanda dos cultivos nessas áreas. Borges et al., (2010) encontraram $3,8 \mathrm{~mm}$. dia ${ }^{-1}$ para o mês de fevereiro pelo método de PM-FAO (56) para o município de Paraipaba, Estado do Ceará. Na Figura 3 (b) também há ocorrência pontuais de áreas com valores mais elevados de ETo, acima de 2,38 mm.dia ${ }^{-1}$, na Região Sudoeste (município de Imperatriz) e Extremo Leste do Estado.

A Figura 2 (a), destaca a distribuição espacial da ETo para o mês de março, onde observa-se uma redução acentuada da ETo na maior parte do Estado, apresentando extensas áreas com valores de ETo abaixo de 2,09 mm. $\mathrm{dia}^{-1}$. Com ocorrências pontuais de áreas com valores mais elevados de ETo no Leste maranhense (Região do município de Chapadinha), Sudoeste (Região município de Imperatriz) e Extremo Sul do Maranhão, em contrapartida, áreas de valores mínimos encontram-se na maior parte da região Oeste e nas regiões Baixadas Litorâneas (Região de São Luís, Alcântra, Cururupu e Turiçu), Bacabal na 
região central e em porções das regiões Sudeste e Sul do Estado.

A Figura 2 (b) apresenta a distribuição espacial distribuição espacial da ETo para o mês de abril. Nota-se que a estação chuvosa na Região Norte se acentua e juntamente com a influência da alta umidade relativa do ar da Região Litorânea faz com que a ETo seja menor em boa parte da Região Norte e Oeste maranhense, assim as médias da ETo para o mês abril aumentam de forma gradativa ao se afastar do oceano em direção ao Centro-Sul do Estado. Entretanto, os valores mais elevados destas áreas estão localizados na Região extremo Sul do Estado e em áreas pontuais no Leste, extremo Leste (município de Araioses) e Sudoeste Maranhense.

A Figura 3 (a) apresenta a distribuição espacial distribuição espacial da ETo referente ao mês de maio que aumenta de forma gradativa ao se afastar do oceano em direção ao Sul do Estado. Assim verifica-se que as áreas da Baixada Litorânea correspondente a Região Metropolitana de São Luís e dos municípios Icatu, Rosário, Itapecuru Mirim, Matinha, Pinheiro e Alcântara apresentam os menores valores de ETo para o mês, sendo inferiores a 2,33 mm. dia ${ }^{-1}$, resultado atribuído a continuidade do período chuvoso e a maior umidade relativa do ar nessa região. Os valores mais elevados começam a ocorrer pontualmente em áreas do Sudoeste (município de Imperatriz), e no município de Balsas no Sul do Maranhão, caracterizando o início da estação seca no Sul do Estado.

Encontra-se na Figura 3 (b) a espacialização das médias diárias na escala mensal da ETo, obtidas para o mês de junho, em que novamente se observa uma grande influência da estação chuvosa e da umidade relativa do ar dessas áreas costeiras que faz com que a ETo seja menor em extensas áreas da Região Norte, logo como se depreende da Figura 3 (b) que a espacialização da ETo para o mês de junho demonstra que a média aumenta de forma gradativa ao se afastar do oceano em direção ao Sul do Estado, com ocorrências pontuais de áreas com valores mais elevados de ETo no Oeste, Leste, e Extremo Sul maranhense.
Como se depreende da Figura 4 os meses de julho (Figura 4 a) e agosto (Figura 4 b) apresentam padrão de distribuição espacial da ETo bem semelhante, onde se observa uma grande influência da estação chuvosa no Norte e a umidade relativa do ar das áreas costeiras que faz com que a ETo seja menor na Região Norte, aumentando de forma gradativa ao se afastar do oceano em direção ao Centro-Sul do Estado. Com ocorrências pontuais de áreas com valores mais elevados de ETo no Leste maranhense (Regiões de divisa com Piauí) e Sul (Região do município de Porto Franco). Como se observa na Figura 4 (b) o mês de agosto apresenta um aumento significativo na ETo em comparação com os meses anteriores, devido a intensificação da estação seca no Sudoeste e Sul do Estado do Maranhão, que vai de maio a novembro, portanto a partir deste mês começam a aparecer nessas Regiões áreas pontuais apresentando demanda hídrica acima de 3,99 mm. dia ${ }^{-1}$.

A Figura 5 (a) apresenta a distribuição espacial distribuição espacial da ETo referente ao mês de setembro que aumenta de forma gradativa de Oeste para Leste (Regiões de divisa com Piauí) e a estação seca se intensifica no Estado do Maranhão o que contribui para a ocorrência de extensas áreas, com valores elevados de ETo acima de 4,2 mm. dia $^{-1}$, assim a estação seca que se inicia no mês de maio atinge o seu pico de setembro. De acordo com Shen et al., (2013) identificar focos e picos de demanda de água é importante para atender os cultivos irrigados, informações essenciais para o planejamento e operação de sistemas de irrigação, sobretudo quanto se busca a otimização dos recursos hídricos disponíveis (AZHAR; PERERA, 2011). Nota-se também, que na distribuição espacial da ETo para o mês de setembro há uma nítida redução da área de influência da alta umidade relativa do ar da Região Litorânea que faz com que a ETo seja menor na Região Norte do Estado, em comparação com os meses anteriores.

Como se observa na Figura 5 (b), que corresponde ao mês de outubro há um aumento significativo de áreas com valores elevados de ETo, acima de 3,46 mm. dia ${ }^{-1}$, no Leste Maranhense (Regiões de divisa com Piauí) que se estendem em direção ao centro do Estado e 
mais redução da área de influência da precipitação e da alta umidade relativa do ar na ETo das Regiões Litorâneas, caracterizando o início da estação seca no Norte do Estado. Entretanto, valores de ETo abaixo de 3,08 mm. dia $^{-1}$, também ocorrem pontualmente em parte do Oeste, Sul e Norte maranhense

A Figura 6 (a) apresenta a distribuição espacial distribuição espacial da ETo para o mês de novembro. Nota-se que a estação seca na Região Norte se acentua, juntamente com significativa redução da área de influência da alta umidade relativa do ar na Região Litorânea ficando limitada apenas as áreas da Baixada correspondente a Região Metropolitana de São Luís. O mês de novembro corresponde o período de estação seca na Região Norte e Leste do Estado e início da estação chuvosa na Região Sudoeste e Sul do Maranhão. O início do período chuvoso no Sudoeste e Sul contribui para a ocorrência de áreas com valores de ETo abaixo de 2,32 mm. dia ${ }^{-1}$, enquanto que em boa parte da Região Norte e Leste predominam a ocorrência de áreas com demanda hídrica acima de 3,28 mm. dia ${ }^{-1}$.

Como se depreende na análise da Figura 6 (b) o mês de dezembro apresenta padrão de distribuição espacial da ETo bem semelhante ao mês de novembro, onde se observa que os valores mais elevados de ETo se concentram no Norte e Leste do Estado com ocorrência de maior parte das áreas apresentando valores de ETo acima de 2,65 mm. dia $^{-1}$, enquanto que Região Sudoeste e Sul do Maranhão predominam a ocorrência de áreas com demanda hídrica abaixo de 2,41 mm. dia-1 .

A distribuição da variável em mapas interpolados permitiu obter noções da caracterização do Estado do Maranhão em relação ao comportamento anual da ETo.

O trimestre mais seco é formado pelos meses de agosto, setembro e outubro e as médias mensais de ETo variaram de $1,95 \mathrm{~mm}$. dia ${ }^{-1}$ (fevereiro) à 3,82 mm. dia ${ }^{-1}$ (setembro).

A região Leste (na divisa com Piauí) é a que apresenta maior de déficit hídrico ao longo do ano, logo o uso da irrigação é fundamental para se obter boa produção agrícola, devendo tomar maiores cuidados na estimativa da ETo na elaboração e manejo de projetos de irrigação.
Percebe-se que nas Regiões Sudoeste e Sul a ocorrência de maiores valores de ETo nos meses de maio a setembro caracterizando o pico da estação seca nessas regiões. Já período que vai de outubro a fevereiro é caracterizado por uma clara inversão de padrões de ETo ocorrendo o pico da estação seca nas Regiões Norte e Leste do Maranhão.

Silva et al., (2013) trabalharam com a espacialização da Evapotranspiração de referência (ETo) para o Estado do Ceará utilizando o método IDW e obtiveram bom desempenho do interpolador que se mostrou uma ferramenta adequada para a espacialização da variável em estudo. Gardiman Junior et al., (2012), Magalhães et al., (2013) e Amorim et al., (2008) também obtiveram bom desempenho na utilização do método IDW como ferramenta SIG para a realização de interpolações de valores de precipitação pluvial. Entretanto, de acordo com Saraiva et al., (2017) ainda não há um método de interpolação considerado como "o mais adequado” para estimativa e espacialização de variáveis meteorológicas como a evapotranspiração

Os mapas interpolados de Evapotranspiração facilitaram a análise temporal e espacial tornando-a clara e objetiva e disponibilizará aos produtores informações que poderão ser úteis na definição estratégias mais eficientes no manejo da irrigação durante o ano em cada região, enquanto não existem os valores locais medidos por métodos diretos, além disso o profissional e o produtor poderão escolher e dimensionar melhor um projeto de irrigação para o período de maior estiagem e demanda hídrica, variando de acordo com a época do ano e o tipo da cultura através da observação e comparação dos mapas climáticos.

\section{CONCLUSÕES}

1- No período avaliado o mês de março e setembro se caracterizaram respectivamente como os meses de menor e maior demanda hídrica no Estado do Maranhão.

2- A região da Baixada Litorânea correspondente a Região Metropolitana de São Luís, apresentou a menor evapotranspiração de 
referência média para o período avaliado o que pode ser atribuído a influência da alta umidade relativa do ar dessas áreas costeiras.

3- A distribuição da variável em mapas interpolados permitiu obter noções da caracterização do Estado do Maranhão em relação ao comportamento anual da ETo, fornecendo informações úteis sobre o comportamento anual da ETo e para o manejo da irrigação em distintas microrregiões do Estado.

\section{REFERÊNCIAS BIBLIOGRÁFICAS}

ALLEN, R. G.; PEREIRA, L. S.; RAES, D.; SMITH, M. Crop evapotranpiration: Guidelines for computing crop water requirements. Rome: FAO, 1998. 301p. Irrigation and Drainage, Paper 56.

AMORIM, R. C. F.; RIBEIRO, A.; LEITE, C. C.; LEAL, B. G.; SILVA, J. B. G. Avaliação do desempenho de dois métodos de espacialização da precipitação pluvial para o estado de Alagoas. Acta Scientiarum Technology, v. 30, n. 1, p. 87-91, 2008.

AZHAR, A. H.; PERERA, B. J. C. Evaluation of Reference Evapotranspiration Estimation Methods under Southeast Australian Conditions. Journal of Irrigation and Drainage Engineering, v.137, n.5, p.268-279, 2011.

BERNARDO, S.; SOARES, A. A. ; MONTOVANI,.E.C. Manual de irrigação. 8 ed Viçosa, MG; UFLA, 2005. 625p.

BORGES, R. L. M.; AZEVEDO, B. M.; CAMPÊLO, A. R.; PINHEIRO NETO,L. G.; VIANA,T. V. A. Evapotranspiração de referência utilizando a equação de PenmanMonteith com diferentes metodologias para o cálculo da temperatura média diária do ar. Agropecuária Técnica, v. 31, n. 1, 2010.

CARVALHO, D. F.; CRUZ, E. S.; SILVA, W. A.; SOUZA, W. J.; SOBRINHO, T. A.
Demanda hídrica do milho de cultivo de inverno no Estado do Rio de Janeiro. Revista

Brasileira de Engenharia Agrícola e Ambiental, v.10, n.1, p.112-118, jan/mar. 2006. Disponível em:< http://www.scielo.br/pdf/rbeaa/v10n1/v10n1a1 7.pdf>. Acesso em: 07 abr. 2016. DOI: http://dx.doi.org/10.1590/S141543662006000100017.

CARVALHO, L. G.; RIOS, G. F. A.; MIRANDA, W. L.; NETO, P. C. Evapotranspiração de referência: uma abordagem atual de diferentes métodos de estimativa. Pesquisa Agropecúaria Tropical., Goiânia, v. 41, n. 3, p. 456-465, jul./set. 2011.

FARIA, Manoel Teixeira et al. Resposta produtiva do feijoeiro comum a diferentes manejos de irrigação. Irriga, v. 17, n. 2, p. 137, 2012.

GARDIMAN JUNIOR, B. S.; MAGALHÃES, I. A. L.; FREITAS, C. A. A.; CECÍLIO, R. A. Análise de técnicas de interpolação para espacialização da precipitação pluvial na bacia do rio Itapemirim (ES). Revista do Setor de Ciências Agrárias e Ambientais, v. 8, n. 1. p. 61-71, 2012.

LI, J.; HEAP, A. A review of comparative studies of spatial interpolation methods in environmental science: performance and impact factors. Ecological Informatics, v. 6, p. 228241, 2011.

MAGALHÃES, I. A. L.; ALMEIDA, K. L.; THIAGO, C. R. L.; GARDIMAN JUNIOR, B. S.; ZANETTI, S. S.; CECÍLIO, R. A. Análise de métodos de interpolação para espacialização da precipitação pluvial na região norte do estado do Espírito Santo. In: Anais. XVI Simpósio Brasileiro de Sensoriamento Remoto - SBSR, Foz do Iguaçu, Paraná, 2013.

MARTÍ, P.; ZARZO, M.; VANDERLINDEN, K.; GIRONA, J. Parametric expressions for the adjusted Hargreaves coefficient in Eastern Spain. Journal of Hydrology, 2015. 
MENESES, L.F.; NASCIMENTO, M.A.L.; Sistemas de informação geográfica aplicados à elaboração de frameworks de geodiversidade. Revista Estudos Geoambientais, n.1, v.1; jan/abr. 2014. Disponível em:< http://periodicos.ufpb.br/index.php/geo/article/ view/19128>. Acesso em: 07 abr. 2016.

PELLEGRINO, G. Q.; PINTO, H. S.; ZULLO JÚNIOR, J.; BRUNINI, O. O uso de sistemas de informações geográficas no mapeamento de informações agrometeorológicas. In: ASSAD, E. D.; SANO, E. E. Sistemas de informações Geográficas aplicações na agricultura. Brasília: Embrapa-SPI / EmbrapaCPAC, 1998.

RÁCZ， C.; NAGY, J.; DOBOS, A. C. Comparison of Several Methods for Calculation of Reference Evapotranspiration. Acta Silvatica \& Lignaria Hungarica, v.9, p.9-24, 2013.

SARAIVA,G. S.; BONOMO, R.; SOUZA, J. M. Avaliação de interpoladores geoestatísticos e determinísticos da evapotranspiração de referência diária para o estado do Espírito Santo. Revista Agro@mbiente On-line, v. 11, n. 1, p. 21-30, janeiro-março, 2017.

SHEN, Y.; LI, S.; CHEN, Y.; QI, Y.; ZHANG, S. Estimation of regional irrigation water requirement and water supply risk in the arid region of Northwestern China 1989-2010. Agricultural Water Management, v.128, p.55-64, 2013.

SILVA, B. B. da; BRAGA, A. C.; BRAGA, C. C.; OLIVEIRA, L. M. M. de; GALVÍNCIO, J. D.; MONTENEGRO, S. M. G. L. Evapotranspiração e estimativa da água consumida em perímetro irrigado do Semiárido brasileiro por sensoriamento remoto. Pesquisa Agropecuára Brasileira v.47, n.9, p.12181226, 2012.
SILVA, M. G.; ARRAES, F. D. D; LEDO, E. R. F. , SANTOS, N. T. , SILVA FILHO, J. A. Avaliação da evapotranspiração de referência por Penman-Monteith usando dados climáticos mínimos no sertão do Ceará. Revista Agro@mbiente On-line, v. 7, n. 3, p. 284-293, setembro-dezembro, 2013.

SOUZA, A. P.; ALMEIDA, F. T.; ARANTES, K. R.; MARTIM, C. C.; SILVA, J. O. Coeficientes de Tanque Classe A para estimativa da evapotranspiração de referência diária na região de transição CerradoAmazônica. Scientia Plena v11, n050204. 2015.

TABARI, H.; GRISMER, M. E.; TRAJKOVIC, $\mathrm{S}$. Comparative analysis of 31 reference evapotranspiration methods under humid conditions. Irrigation Science, v.31, p.107117, 2013.

TAGLIAFERRE, C.; DE PAULA, A.; ROCHA, F. A.; CAMPOS, W. V.; GUIMARÃES, D. U. Influência dos elementos meteorológicos na evapotranspiração de referência estimada utilizando-se o irrigâmetro no município de Guanambi-BA. Revista Engenharia na Agricultura, v. 23, n. 3, p. 251, 2015.

USMAN, M.; LIEDL, R.; AWAN, U. K. Spatiotemporal estimation of consumptive water use for assessment of irrigation system performance and management of water resources in irrigated Indus Basin, Pakistan. Journal of Hydrology, v.525, p.26-41, 2015.

VILANOVA, M. R. N.; SIMÕES, S. J. C.; TRANNIN, I. C, B. Interpolação geoespacial da evapotranspiração de referência (ETo) em regiões com escassez de dados: estudo de caso no Sul de Minas Gerais. Ambiente e Agua, Taubaté, v. 7, n. 2, p. 179194, 2012. 